EPJ manuscript No.

(will be inserted by the editor)

\title{
Yukawa Coupling Structure in Intersecting D-brane Models
}

\author{
Noriaki Kitazawa ${ }^{1}$, Tatsuo Kobayashi ${ }^{2}$, Nobuhito $\mathrm{Maru}^{3}$, and Nobuchika Okada ${ }^{4}$ \\ 1 Department of Physics, Tokyo Metropolitan University, Hachioji, Tokyo 192-0397, Japan \\ e-mail: kitazawa@phys.metro-u.ac.jp \\ 2 Department of Physics, Kyoto University, Kyoto 606-8502, Japan \\ e-mail: kobayash@gauge.scphys.kyoto-u.ac.jp \\ 3 Theoretical Physics Laboratory, RIKEN, 2-1 Hirosawa, Wako, Saitama 351-0198, Japan \\ e-mail: maru@postman.riken.go.jp \\ 4 Theory Group, KEK, 1-1 Oho, Tsukuba, Ibaraki 305-0801, Japan \\ e-mail: okadan@post.kek.jp
}

\begin{abstract}
The structure of Yukawa coupling matrices is investigated in type IIA $T^{6} /\left(\mathrm{Z}_{2} \times \mathrm{Z}_{2}\right)$ orientifold models with intersecting D-branes. Yukawa coupling matrices are difficult to be realistic in the conventional models in which the generation structure emerges by the multiple intersection of D-branes in the factorized $T^{6}=T^{2} \times T^{2} \times T^{2}$. We study the new type of flavor structure, where Yukawa couplings are dynamically generated, and show this type of models lead to nontrivial structures of Yukawa coupling matrices, which can be realistic.
\end{abstract}

\section{Introduction}

Understanding the masses and flavor mixing of quarks and leptons is one of the most important issues in particle physics. New physics which derive Yukawa coupling matrices in the standard model should certainly exist. Yukawa couplings, in a sense, seem naturally of $\mathcal{O}(1)$. Thus, how to derive suppressed Yukawa couplings is a key to under'stand the hierarchy of fermion masses and mixing angles.

String theory, which is a candidate of the consistent theory of quantum gravity, gives an attractive framework to generate Yukawa coupling matrices. Indeed, in several types of string models coupling selection rules have been studied and Yukawa couplings have been calculated. Recent development of the models with intersecting Dbranes, intersecting D-brane models (see Refs. 1, 2,3 4 for essential idea), opens a new possibility towards realistic models. Among them, models with low-energy supersymmetry [4, 5, 6, 7, 8, 9, 10, 11, 12, 13, 14, 15] are interesting, because those are constructed as stable solutions of the string theory. Within the framework of intersecting Dbrane models, open string modes corresponding to matter fields and Higgs fields are localized at intersecting points between D-branes [16]. The flavor number is obtained as intersecting numbers of D-branes in conventional models.

Intersecting D-brane models have several interesting aspects from phenomenological viewpoints. In particular, their Yukawa couplings have a phenomenologically important feature. Calculation of Yukawa couplings in intersecting D-brane models [17, 18, 19, 20,21] is similar to Yukawa calculation of twisted strings in heterotic orbifold models
22 23 24 ${ }^{1}$. These Yukawa couplings in both intersecting D-brane models and heterotic orbifold models depend on localized points of matter modes and moduli corresponding to the volume of compact space. Thus, Yukawa couplings are, in general, non-universal, and can lead to suppressed values. Indeed, heterotic orbifold models have possibilities for realizing realistic Yukawa matrices [25]. Also intersecting D-brane models have the potential to lead to realistic Yukawa matrices, but most of intersecting D-brane models, which have been constructed so far, seem to lead to the factorizable form of Yukawa matrices, $y_{i j}=a_{i} b_{j}$, that is, rank-one matrices. With this form of Yukawa matrices, one can derive only a non-vanishing mass for the third family, but vanishing values for other lighter masses and mixing angles [26. Hence, it is quite important to study a new type of flavor structure which can lead to non-vanishing mixing angles and light fermion masses within the framework of intersecting D-brane models.

In Ref. [15] one of the authors has proposed an intersecting D-brane model with a new type of flavor structure, where quarks, leptons and Higgs doublets appear as composite fields. In this model, Yukawa couplings are dynamically generated. Since the origin of flavors is different from the one in the conventional models, the structure of Yukawa coupling matrices can be different from the one in conventional models. In this paper we investigate the structure of Yukawa coupling matrices of the model, and show that the nontrivial form of Yukawa matrices is ob-

\footnotetext{
1 Yukawa couplings in heterotic models are calculable, because string is solvable on orbifolds.
} 
Noriaki Kitazawa et al.: Yukawa Coupling Structure in Intersecting D-brane Models

tained in this framework of Yukawa coupling generations, e.g. non-vanishing mixing angles. Thus, our scenario is interesting to realize realistic values of fermion masses and mixing angles.

This paper is organized as follows. In section [2] we give a brief review on intersecting D-brane models. Then, we discuss the structure of Yukawa coupling matrices in conventional models. We point out that it is difficult to have realistic structure in general. In section 3 we briefly introduce a model of dynamical generation of Yukawa couplings which is proposed in Ref. 15. In section 4 a detailed analysis of the structure of Yukawa coupling matrices is given. We show that our framework of the Yukawa coupling generation derives a nontrivial structure of Yukawa matrices, which can be realistic. Section 5 is devoted to conclusions and discussions.

\section{Intersecting D-brane models and Yukawa couplings}

\subsection{Intersecting D-brane models}

Here we give a brief review on intersecting D-brane models. Consider the type IIA superstring theory compactified on $\mathbf{T}^{6} /\left(\mathbf{Z}_{2} \times \mathbf{Z}_{2}\right)$ orientifold, where $\mathbf{T}^{\mathbf{6}}=\mathbf{T}^{\mathbf{2}} \times \mathbf{T}^{\mathbf{2}} \times \mathbf{T}^{\mathbf{2}}$. The type IIA theory is invariant under the $\mathbf{Z}_{\mathbf{2}} \times \mathbf{Z}_{\mathbf{2}}$ transformation

$$
\begin{aligned}
\theta: & X_{ \pm}^{k} \rightarrow e^{ \pm i 2 \pi v_{k}} X_{ \pm}^{k}, \\
\omega: & X_{ \pm}^{k} \rightarrow e^{ \pm i 2 \pi w_{k}} X_{ \pm}^{k},
\end{aligned}
$$

where $v=(0,0,1 / 2,-1 / 2,0)$ and $w=(0,0,0,1 / 2,-1 / 2)$ and

$$
X_{ \pm}^{k}= \begin{cases}\frac{1}{\sqrt{2}}\left( \pm X^{2 k}+X^{2 k+1}\right), & \text { for } k=0, \\ \frac{1}{\sqrt{2}}\left(X^{2 k} \pm i X^{2 k+1}\right), & \text { for } k=1,2,3,4\end{cases}
$$

with space-time coordinates $X^{\mu}, \mu=0,1, \cdots, 9$. The type IIA theory is also invariant under the $\Omega R$ transformation, where $\Omega$ is the world-sheet parity transformation and

$$
R: \quad \begin{cases}X^{i} \rightarrow X^{i}, & \text { for } i=0,1,2,3,4,6,8, \\ X^{j} \rightarrow-X^{j}, & \text { for } j=5,7,9 .\end{cases}
$$

We mod out the theory by the action of $\theta, \omega, \Omega R$ and their independent combinations.

A D6 $6_{a}$-brane stretching over our three-dimensional space and winding in compact $\mathbf{T}^{\mathbf{2}} \times \mathbf{T}^{\mathbf{2}} \times \mathbf{T}^{\mathbf{2}}$ space is specified by the winding numbers in each torus:

$$
\left[\left(n_{a}^{1}, m_{a}^{1}\right),\left(n_{a}^{2}, m_{a}^{2}\right),\left(n_{a}^{3}, m_{a}^{3}\right)\right] .
$$

A D6 $6_{a}$-brane is always accompanied by its orientifold image $\mathrm{D} 6_{a^{\prime}}$ whose winding numbers are

$$
\left[\left(n_{a}^{1},-m_{a}^{1}\right),\left(n_{a}^{2},-m_{a}^{2}\right),\left(n_{a}^{3},-m_{a}^{3}\right)\right] .
$$

The number of intersection between D6 $6_{a}$-brane and D6 $b_{b^{-}}$ brane is given by

$$
I_{a b}=\prod_{i=1}^{3}\left(n_{a}^{i} m_{b}^{i}-m_{a}^{i} n_{b}^{i}\right)
$$

\begin{tabular}{|c|l|}
\hline sector & field \\
\hline \hline$a a$ & $\mathrm{U}\left(N_{a} / 2\right)$ or $\operatorname{USp}\left(N_{a}\right)$ gauge multiplet. \\
& $3 \mathrm{U}\left(N_{a} / 2\right)$ adjoint or $3 \operatorname{USp}\left(N_{a}\right)$ \\
& anti-symmetric tensor chiral multiplets. \\
\hline$a b+b a$ & $I_{a b}\left(\square_{a}, \square_{b}\right)$ chiral multiplets. \\
\hline$a b^{\prime}+b^{\prime} a$ & $I_{a b^{\prime}}\left(\square_{a}, \square_{b}\right)$ chiral multiplets. \\
\hline$a a^{\prime}+a^{\prime} a$ & $\frac{1}{2}\left(I_{a a^{\prime}}-\frac{4}{2^{k}} I_{a O 6}\right)$ symmetric tensor \\
& $\begin{array}{l}\text { chiral multiplets. } \\
\frac{1}{2}\left(I_{a a^{\prime}}+\frac{4}{2^{k}} I_{a O 6}\right) \text { anti-symmetric tensor } \\
\text { chiral multiplets. }\end{array}$ \\
\hline
\end{tabular}

Table 1. General massless field contents on intersecting D6-branes. In $a$ a sector, the gauge symmetry is $\operatorname{USp}\left(N_{a}\right)$ or $\mathrm{U}\left(N_{a} / 2\right)$ corresponding to whether $\mathrm{D} 6_{a}$-brane is parallel or not to some O6-plane, respectively. In $a a^{\prime}+a^{\prime} a$ sector, $k$ is the number of tilted torus, and $I_{a O 6}$ is the sum of the intersection numbers between D6 $6_{a}$-brane and all O6-planes.

The intersecting angles, $\theta_{a}^{i}$ with $i=1,2,3$, between D6 $a^{-}$ brane and $X^{4}, X^{6}$ and $X^{8}$ axes in each torus are given by

$$
\theta_{a}^{i}=\tan ^{-1}\left(\chi_{i} \frac{m_{a}^{i}}{n_{a}^{i}}\right)
$$

where $\chi_{i}$ are the ratios of two radii of each torus: $\chi_{i} \equiv$ $R_{2}^{(i)} / R_{1}^{(i)}$. The system has supersymmetry, if $\theta_{a}^{1}+\theta_{a}^{2}+\theta_{a}^{3}=$ 0 is satisfied for all $a$. The configuration of intersecting D6-branes should satisfy the following Ramond-Ramond tadpole cancellation conditions,

$$
\begin{aligned}
\sum_{a} N_{a} n_{a}^{1} n_{a}^{2} n_{a}^{3} & =16 \\
\sum_{a} N_{a} n_{a}^{1} m_{a}^{2} m_{a}^{3} & =-16 \\
\sum_{a} N_{a} m_{a}^{1} n_{a}^{2} m_{a}^{3} & =-16 \\
\sum_{a} N_{a} m_{a}^{1} m_{a}^{2} n_{a}^{3} & =-16
\end{aligned}
$$

where $N_{a}$ is the multiplicity of $\mathrm{D} 6_{a}$-brane, and we are assuming three rectangular (untilted) tori. The NeveuSchwarz-Neveu-Schwarz tadpoles are automatically cancelled, when Ramond-Ramond tadpole cancellation conditions and the supersymmetry conditions are satisfied.

There are four sectors of open string corresponding to on which D6-branes two ends of open string are fixed: $a a$, $a b+b a, a b^{\prime}+b^{\prime} a, a a^{\prime}+a^{\prime} a$ sectors. Each sector gives matter fields in four-dimensional low-energy effective theory. The general massless field contents are given in Table 1 A common problem of the model building in this framework is the appearance of the massless adjoint fields in $a$ a sector, since there are no massless matter fields in the adjoint representation under the standard model gauge group in Nature. These fields are expected to be massive in the case of a curved compact space, because these are the moduli fields of D6-brane configurations. 


\subsection{The Yukawa Coupling Structure in Conventional Models}

The magnitude of Yukawa couplings among localized open string modes can be calculated by conformal field theory technique like heterotic orbifold models. The important part of 3-point interactions $y$ is evaluated by the classical part as [17, 18, 19

$$
y \sim e^{-S_{c l}} \sim \prod_{i} e^{-\Sigma_{i} / 2 \pi \alpha^{\prime}} .
$$

Here, $S_{c l}$ denotes the action of classical string solution $X_{c l}$, which have the asymptotic behavior corresponding to local open string modes near intersecting points, and $S_{c l}$ is obtained as a product of the triangle areas $\Sigma_{i}$ on the $i$-th $T^{2}$, which string sweeps to couple. These Yukawa couplings can lead to suppressed values when intersecting points are far away each other. Thus, intersecting Dbrane models have the potential to realize the hierarchy of fermion masses and mixing angles.

Here, however, we discuss the difficulty to generate a realistic structure of Yukawa coupling matrices in the models from type IIA $T^{6} /\left(\mathrm{Z}_{2} \times \mathrm{Z}_{2}\right)$ orientifolds with intersecting D-branes. The six-dimensional torus is assumed to be factorizable: $T^{6}=T^{2} \times T^{2} \times T^{2}$.

As an illustrating example, let us consider a toy model with three D6-branes, $\mathrm{D}_{3}$-brane for $\mathrm{SU}(3)_{c}$ gauge symmetry, $\mathrm{D} 6_{2}$-brane for $\mathrm{SU}(2)_{L}$ gauge symmetry and $\mathrm{D} 6_{1}$-brane for one component of $\mathrm{U}(1)_{Y}$ gauge symmetry, and a very simple configuration of intersecting numbers

$$
I_{a b}=\left(\begin{array}{ccc}
0 & 1 & 3 \\
-1 & 0 & 3 \\
-3 & -3 & 0
\end{array}\right)_{a b},
$$

where $a, b=1,2,3$. This system gives three generations of quarks, three left-handed quark doublets and three righthanded quark singlets, and one Higgs doublet with Yukawa couplings. The total intersecting number $I_{a b}$ of $\mathrm{D} 6_{a}$-brane and $\mathrm{D} 6 b_{b}$-brane is given by the multiplications of three intersecting numbers of $\mathrm{D} 6_{a}$-brane and $\mathrm{D} 6_{b}$-brane in each of three tori. The structure of Yukawa coupling is not fully determined by the intersecting number, but it depends on the configuration of the intersection in each of three tori.

The intersecting number $I_{12}=1$ means one Higgs doublet field localizing a point in each of three tori. The intersecting number $I_{13}=3$ means three intersections in one of three tori and one intersection in other two tori. The same is true for $I_{23}=3$. Therefore, we have two different configurations of intersection: (1) D6 $6_{1}$-brane and D6 $6_{3}$-brane intersect three times in a torus in which $\mathrm{D} 6_{2}$-brane and D6 3 -brane also intersect three times, or (2) D6 ${ }_{1}$-brane and D6 3 -brane intersect three times in a torus in which D6 $2^{-}$ brane and $\mathrm{D} 6_{3}$-brane intersect once.

In both cases, Yukawa couplings are evaluated by Eq. (13) The quantum part also contributes to Yukawa couplings, but gives $\mathcal{O}(1)$ of common factor. In case (1) the Yukawa matrix can become hierarchical but always diagonal. In this case $\mathrm{D} 6{ }_{3}$-brane should wind three times in a torus and intersect with each $\mathrm{D} 6_{1}$-brane and $\mathrm{D} 6_{2}$-brane once in each winding. This gives definite three pairs of righthanded and left-handed quarks and defines three generations without mixing. The values of Yukawa couplings are determined by the areas of triangles which are determined by the place of each pair of quarks and Higgs doublet. In this case we can not have flavor mixing. This structure of Yukawa coupling matrix seems unable to be modified by the quantum corrections in supersymmetric models.

In case (2) we always have factorized structure of Yukawa coupling matrix:

$$
y_{i j} \propto a_{i} b_{j},
$$

where $i, j=1,2,3$. In a torus where D6 ${ }_{1}$-brane intersects three times with $\mathrm{D} 6{ }_{3}$-brane three left-handed quarks localize at one point and Higgs doublet also localizes at one point. We have three triangle areas which determine the value of $a_{i}$ or $b_{i}$. The same is true for the torus where D6 $6_{2}$-brane intersects three times with $\mathrm{D} 6_{3}$-brane. Therefore, the Yukawa coupling matrix is the multiplication of the contribution from each three torus. Furthermore, the Kähler metric depends on twisted angles of open string [20]. The twisted angle of open strings corresponding to three left-handed quarks is common, and we also have the same twisted angle for three right-handed quarks. Thus, the Kähler metric is relevant to only the overall magnitude of Yukawa matrices, but irrelevant to ratios of entries in Yukawa matrices. In this case we can not have realistic mass spectrum, since the rank of the Yukawa coupling matrix is one. This structure of Yukawa coupling matrix also seems unable to be modified by the quantum corrections in supersymmetric models.

We have discussed the problem in the simplified model, but most of models seem to have the almost same problem. In particular, it seems difficult to derive non-vanishing mixing angles as well as lighter fermion masses. There are several possibilities to overcome this difficulty.

First, we can try to construct models with some further complications by arranging the configuration of D6branes. For example, introduction of many Higgs doublets may solve the problem [27, though it is not experimentally favored. To have three generations utilizing orientifold image D6-branes may give a way to solve the problem, though we will have relatively many unwanted exotic fields.

The second way is to change the structure of the compactified space. Leaving from the factorizable $T^{6}=T^{2} \times$ $T^{2} \times T^{2}$ to $T^{6}=T^{4} \times T^{2}$, for example, may make the situation completely change. But the physics becomes less intuitive and the analysis becomes much more complicated.

The third way is to change the origin of the generation. This is the way we are going to take in this paper. In the next section, we introduce a model in which the generation is not originated from the multiple intersection of D6-branes. 


\begin{tabular}{|c|c|c|}
\hline D6-brane & winding number & multiplicity \\
\hline \hline $\mathrm{D}_{1}$ & {$[(1,-1),(1,1),(1,0)]$} & 4 \\
\hline $\mathrm{D}_{2}$ & {$[(1,1),(1,0),(1,-1)]$} & $6+2$ \\
\hline $\mathrm{D}_{3}$ & {$[(1,0),(1,-1),(1,1)]$} & $2+2$ \\
\hline $\mathrm{D}_{4}$ & {$[(1,0),(0,1),(0,-1)]$} & 12 \\
\hline $\mathrm{D}_{5}$ & {$[(0,1),(1,0),(0,-1)]$} & 8 \\
\hline D6 $_{6}$ & {$[(0,1),(0,-1),(1,0)]$} & 12 \\
\hline
\end{tabular}

Table 2. Configuration of intersecting D6-branes. All three tori are considered to be rectangular (untilted). Three D6branes, D6 $4, \mathrm{D}_{5}$ and $\mathrm{D} 6_{6}$, are on top of some O6-planes.

\section{A Model of Dynamically Generated Yukawa Couplings}

In this section we give a brief description of the model which has been introduced in Ref. 15.

The D6-brane configuration of the model is given in Table 2 Both tadpole cancellation conditions and supersymmetry conditions are satisfied in this configuration under the conditions of $\chi_{1}=\chi_{2}=\chi_{3} \equiv \chi$. The D6 $2^{-}$ brane system consists of two parallel D6-branes with multiplicities six and two which are separated in the second torus in a consistent way with the orientifold projections. The D6 $6_{3}$-brane system consists of two parallel D6-branes with multiplicity two which are separated in the first torus in a consistent way to the orientifold projections. $\mathrm{D} 6_{1}, \mathrm{D} 6_{2}$ and $\mathrm{D} 6_{3}$ branes give gauge symmetries of $\mathrm{U}(2)_{L}=\mathrm{SU}(2)_{L} \times \mathrm{U}(1)_{L}, \mathrm{U}(3)_{c} \times \mathrm{U}(1)=\mathrm{SU}(3)_{c} \times \mathrm{U}(1)_{c} \times \mathrm{U}(1)$ and $\mathrm{U}(1)_{1} \times \mathrm{U}(1)_{2}$, respectively. The hypercharge is defined as

$$
\frac{Y}{2}=\frac{1}{2}\left(\frac{Q_{c}}{3}-Q\right)+\frac{1}{2}\left(Q_{1}-Q_{2}\right),
$$

where $Q_{c}, Q, Q_{1}$ and $Q_{2}$ are charges of $\mathrm{U}(1)_{c}, \mathrm{U}(1), \mathrm{U}(1)_{1}$ and $\mathrm{U}(1)_{2}$, respectively. The additional non-anomalous $\mathrm{U}(1)$ charge, $Q_{R}$, is defined as

$$
Q_{R}=Q_{1}-Q_{2} .
$$

The remaining three $\mathrm{U}(1)$ gauge symmetries which are generated by $Q_{L}$ (namely $\left.\mathrm{U}(1)_{L}\right), Q_{c}+Q$ and $Q_{1}+Q_{2}$ are anomalous, and their gauge bosons have masses of the order of the string scale. These three anomalous $\mathrm{U}(1)$ gauge symmetries are independent from the two non-anomalous $\mathrm{U}(1)$ gauge symmetries: $\operatorname{tr}\left((Y / 2) Q_{L}\right)=0$, for example.

A schematic picture of the configuration of intersecting D6-branes of this model is given in Fig 1 There are no $a b^{\prime}+b^{\prime} a, a a^{\prime}+a^{\prime} a$ sectors of open string in this configuration. The massless particle contents are given in Table [3. In this table it is assumed that all twelve D6-branes of D6 4 are on top of one of eight O6-branes with the same winding numbers. The same is also assumed for eight and twelve D6-branes of $\mathrm{D} 6_{5}$ and $\mathrm{D} 6_{6}$.

We break three $\mathrm{USp}(12)_{\mathrm{D} 6_{4}}, \mathrm{USp}(8)_{\mathrm{D} 6_{5}}$ and $\mathrm{USp}(12)_{\mathrm{D} 6_{6}}$ gauge symmetries to the factors of $\operatorname{USp}(2)$ gauge symmetries by configuring D6-branes of $\mathrm{D}_{4}, \mathrm{D}_{5}$ and $\mathrm{D} 6_{6}$ as in Fig 2] The resultant gauge symmetries are respectively as

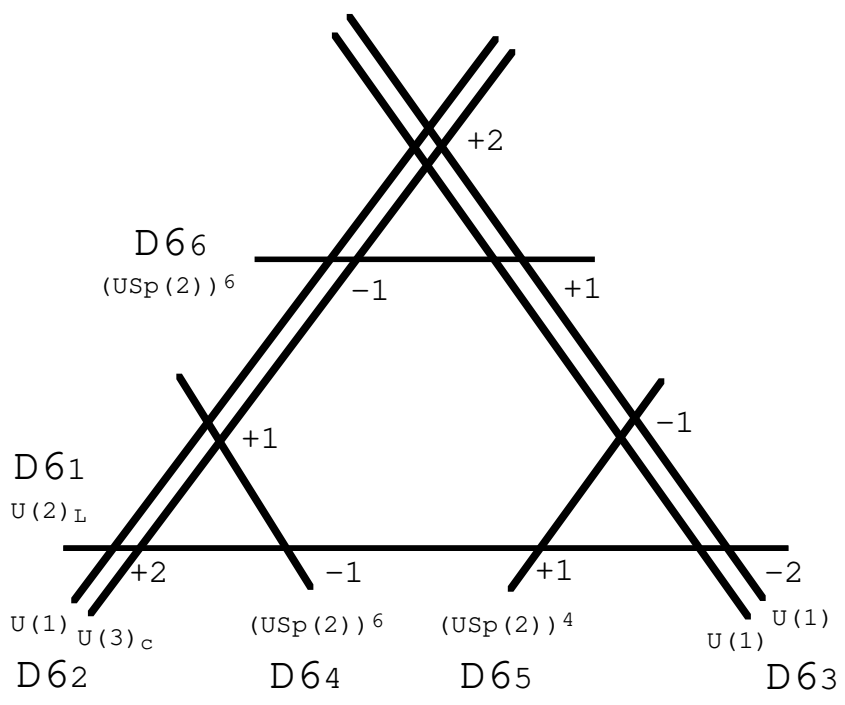

Fig. 1. Schematic picture of the configuration of intersecting D6-branes. This picture describes only the configuration of the intersection of D6-branes each other, and the relative place of each D6-brane has no meaning. The number at the intersection point between $\mathrm{D} 6_{a}$ and $\mathrm{D} 6_{b}$ branes denotes intersection

\begin{tabular}{|c|c|c|}
\hline sector & $\begin{array}{c}\mathrm{SU}(3)_{c} \times \mathrm{SU}(2)_{L} \times \mathrm{USp}(8) \\
\times \mathrm{USp}(12)_{D 6_{4}} \times \mathrm{USp}(12)_{D 6_{6}} \\
\left(Y / 2, Q_{R}\right)\left(Q_{L}, Q_{c}+Q, Q_{1}+Q_{2}\right)\end{array}$ & field \\
\hline$\overline{D 6_{1} \cdot D 6_{2}}$ & $\begin{array}{c}\left.3^{*}, 2,1,1,1\right)_{(-1 / 6,0)(+1,-1,0)} \times 2 \\
(1,2,1,1,1)_{(+1 / 2,0)(+1,-1,0)} \times 2 \\
\end{array}$ & $\overline{\bar{q}_{i}}$ \\
\hline$D 6_{1} \cdot D 6_{4}$ & $(1,2,1,12,1)_{(0,0)(-1,0,0)}$ & $\bar{D}$ \\
\hline$D 6_{2} \cdot D 6_{4}$ & $\begin{array}{l}(3,1,1,12,1)_{(+1 / 6,0)(0,+1,0)} \\
(1,1,1,12,1)_{(-1 / 2,0)(0,+1,0)}\end{array}$ & $\begin{array}{l}C \\
N\end{array}$ \\
\hline$D 6_{1} \cdot D 6_{3}$ & $\begin{array}{l}(1,2,1,1,1)_{(+1 / 2,+1)(-1,0,+1)} \times 2 \\
(1,2,1,1,1)_{(-1 / 2,-1)(-1,0,+1)} \times 2\end{array}$ & $\begin{array}{l}H_{i}^{(1)} \\
\bar{H}_{i}^{(2)} \\
\end{array}$ \\
\hline$D 6_{1} \cdot D 6_{5}$ & $(1,2,8,1,1)_{(0,0)(+1,0,0)}$ & $T$ \\
\hline$D 6_{3} \cdot D 6_{5}$ & $\begin{array}{l}(1,1,8,1,1)_{(+1 / 2,+1)(0,0,-1)} \\
(1,1,8,1,1)_{(-1 / 2,-1)(0,0,-1)}\end{array}$ & $\begin{array}{l}T^{(+)} \\
T^{(-)}\end{array}$ \\
\hline$\overline{D 6_{2} \cdot D 6_{3}}$ & $\begin{array}{c}(3,1,1,1,1)_{(-1 / 3,-1)(0,+1,-1)} \times 2 \\
(3,1,1,1,1)_{(+2 / 3,+1)(0,+1,-1)} \times 2 \\
(1,1,1,1,1)_{(-1,-1)(0,+1,-1)} \times 2 \\
(1,1,1,1,1)_{(0,+1)(0,+1,-1)} \times 2\end{array}$ & $\begin{array}{l}d_{i} \\
\bar{u}_{i} \\
\bar{e}_{i} \\
\bar{\nu}_{i}\end{array}$ \\
\hline$\overline{D 6_{2} \cdot D 6_{6}}$ & $\begin{array}{c}\left(3^{*}, 1,1,1,12\right)_{(-1 / 6,0)(0,-1,0)} \\
(1,1,1,1,12)_{(+1 / 2,0)(0,-1,0)}\end{array}$ & $\begin{array}{l}C \\
\bar{N}\end{array}$ \\
\hline$D 6_{3} \cdot D 6_{6}$ & $\begin{array}{l}(1,1,1,1,12)_{(+1 / 2,+1)(0,0,+1)} \\
(1,1,1,1,12)_{(-1 / 2,-1)(0,0,+1)}\end{array}$ & $\begin{array}{l}\bar{D}^{(+)} \\
\bar{D}^{(-)}\end{array}$ \\
\hline
\end{tabular}
number $I_{a b}$ with $a<b$.

Table 3. Low-energy particle contents before "hypercolor" confinement. The fields from $a a$ sectors are neglected for simplicity.

follows.

$$
\begin{aligned}
& \mathrm{USp}(12)_{\mathrm{D} 6_{4}} \longrightarrow \underset{\alpha=1}{\otimes} \mathrm{USp}(2)_{\mathrm{D} 6_{4}, \alpha}, \\
& \mathrm{USp}(8)_{\mathrm{D} 6_{5}} \longrightarrow \stackrel{4}{\otimes} \mathrm{USp}(2)_{\mathrm{D} 6_{5}, a}, \\
& \mathrm{USp}(12)_{\mathrm{D} 6_{6}} \longrightarrow \stackrel{\otimes}{\alpha=1}_{\alpha} \mathrm{USp}(2)_{\mathrm{D} 6_{6}, \alpha} .
\end{aligned}
$$



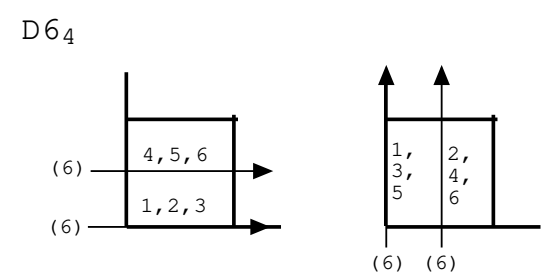

$\mathrm{D} 6_{5}$
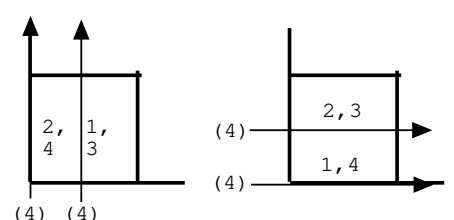

D 66
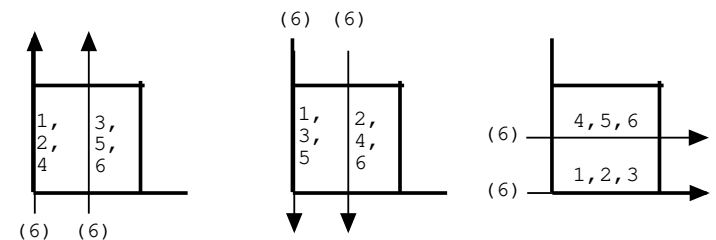

Fig. 2. Configurations of twelve, eight and twelve D6-branes of $\mathrm{D} 6_{4}, \mathrm{D} 6_{5}$ and $\mathrm{D} 6_{6}$ branes, respectively. The numbers in brackets are multiplicities of D6-brane stacks, and the numbers without brackets specify one of the $\operatorname{USp}(2)$ gauge groups in Eqs. (18), (19) and (20). See footnote for details.

All of these USp(2) gauge intersections can be naturally stronger than any other unitary gauge interactions. If we choose $\kappa_{4} M_{s} \sim 1$ and $\chi \sim 0.1$, where $\kappa_{4}=\sqrt{8 \pi G_{N}}$, $M_{s}=1 / \sqrt{\alpha^{\prime}}$, the dynamical scales of all $\operatorname{USp}(2)$ gauge interactions are of the order of $M_{s}$, and the values of the standard model gauge coupling constants are reasonably of $\mathcal{O}(0.01)$ at the string scale. We call these strong $\operatorname{USp}(2)$ interactions "hypercolor" interactions. ${ }^{2}$

The field content in the intersecting $\mathrm{D} 6_{1}-\mathrm{D} 6_{2}-\mathrm{D} 6_{4}$ sector (left-handed sector) is given in Table 4 The confinement of six $\operatorname{USp}(2)_{\mathrm{D} 6_{4}, \alpha}$ gauge interactions gives six generations of left-handed quark and lepton doublets:

$$
C_{\alpha} D_{\alpha} \sim q_{\alpha}, \quad N_{\alpha} D_{\alpha} \sim l_{\alpha},
$$

where $\alpha=1,2, \cdots, 6$. Two of these six left-handed quark doublets and two of these six left-handed lepton doublets

${ }^{2}$ We have many vector-like matters in the configuration of Fig.2, since, for example, D6-branes of D64 and D6 6 overlap in the third torus and intersect in other tori. These vector-like matters spoil asymptotic freedom of "hypercolor" dynamics. But, these vector-like matters get masses of Planck scale, if we move four D-branes of each $\operatorname{USp}(2)$ (two D-branes and their images) a little away from orientifold planes in one torus in a consistent way with orientifold projections and avoid overlapping. This procedure does not change the gauge symmetry and chiral matter contents of the model. In the estimation of the Yukawa couplings, we do not consider this fine structure of the D-brane configuration, since it does not change our main results.

\begin{tabular}{|c|c|c|}
\hline sector & $\begin{array}{c}\left(\mathrm{SU}(3)_{c} \times \mathrm{SU}(2)_{L}\right) \times\left(\mathrm{USp}(2)_{1} \times \mathrm{USp}(2)_{2} \times\right. \\
\left.\mathrm{USp}(2)_{3} \times \mathrm{USp}(2)_{4} \times \mathrm{USp}(2)_{5} \times \mathrm{USp}(2)_{6}\right)_{D 6_{4}} \\
\left(Y / 2, Q_{R}\right)\left(Q_{L}, Q_{c}+Q, Q_{1}+Q_{2}\right)\end{array}$ & field \\
\hline $\bar{D} D 6_{1} \cdot D 6_{2}$ & $\begin{array}{c}\left.3^{*}, 2\right)(1,1,1,1,1,1)_{(-1 / 6,0)(+1,-1,0)} \times 2 \\
(1,2)(1,1,1,1,1,1)_{(+1 / 2,0)(+1,-1,0)} \times 2\end{array}$ & $\begin{array}{c}\bar{q}_{i} \\
\bar{l}_{i}\end{array}$ \\
\hline$D 6_{1} \cdot D 6_{4}$ & $\begin{array}{l}(1,2)(2,1,1,1,1,1)_{(0,0)(-1,0,0)} \\
(1,2)(1,2,1,1,1,1)_{(0,0)(-1,0,0)} \\
(1,2)(1,1,2,1,1,1)_{(0,0)(-1,0,0)} \\
(1,2)(1,1,1,2,1,1)_{(0,0)(-1,0,0)} \\
(1,2)(1,1,1,1,2,1)_{(0,0)(-1,0,0)} \\
(1,2)(1,1,1,1,1,2)_{(0,0)(-1,0,0)}\end{array}$ & $D_{\alpha}$ \\
\hline$D 6_{2} \cdot D 6_{4}$ & $\begin{array}{l}\bar{l}_{(3,1)(2,1,1,1,1,1)_{(+1 / 6,0)(0,+1,0)}} \\
(3,1)(1,2,1,1,1,1)_{(+1 / 6,0)(0,+1,0)} \\
(3,1)(1,1,2,1,1,1)_{(+1 / 6,0)(0,+1,0)} \\
(3,1)(1,1,1,2,1,1)_{(+1 / 6,0)(0,+1,0)} \\
(3,1)(1,1,1,1,2,1)_{(+1 / 6,0)(0,+1,0)} \\
(3,1)(1,1,1,1,1,2)_{(+1 / 6,0)(0,+1,0)} \\
(1,1)(2,1,1,1,1,1)_{(-1 / 2,0)(0,+1,0)} \\
(1,1)(1,2,1,1,1,1)_{(-1 / 2,0)(0,+1,0)} \\
(1,1)(1,1,2,1,1,1)_{(-1 / 2,0)(0,+1,0)} \\
(1,1)(1,1,1,2,1,1)_{(-1 / 2,0)(0,+1,0)} \\
(1,1)(1,1,1,1,2,1)_{(-1 / 2,0)(0,+1,0)} \\
(1,1)(1,1,1,1,1,2)_{(-1 / 2,0)(0,+1,0)}\end{array}$ & $N_{\alpha}$ \\
\hline
\end{tabular}

Table 4. Field contents of the left-handed sector. Here, $i=$ 1,2 and $\alpha=1,2, \cdots, 6$.

become massive through the string-level Yukawa interactions of

$$
W_{\text {left }}=\sum_{i, \alpha} g_{i \alpha}^{\text {left }-\mathrm{q}} \bar{q}_{i} C_{\alpha} D_{\alpha}+\sum_{i, \alpha} g_{i \alpha}^{\text {left-1 }} \bar{l}_{i} N_{\alpha} D_{\alpha}
$$

where $i=1,2$. We have four massless generations of lefthanded quark and lepton doublets. The values of masses are given as $g_{i \alpha}^{\text {left }-\mathrm{q}} \Lambda_{L}$ and $g_{i \alpha}^{\text {left-1 }} \Lambda_{L}$, where $\Lambda_{L}$ denotes the dynamical scale of $\operatorname{USp}(2)_{\mathrm{D} 6_{4}, \alpha}$. The detailed structure of the Yukawa coupling matrix $g_{i \alpha}^{\text {left }-\mathrm{q}}$ is discussed in the next section. Exactly the same happens in the intersecting $D 6_{2^{-}}$ D6 $6_{3}-\mathrm{D} 6_{6}$ sector (right-handed sector). The confinement of six $\operatorname{USp}(2)_{\mathrm{D}_{6}, \alpha}$ gauge interactions gives six generations of right-handed quarks and leptons:

$$
\begin{array}{rlrl}
\bar{C}_{\alpha} \bar{D}_{\alpha}^{(-)} & \sim u_{\alpha}, & & \bar{C}_{\alpha} \bar{D}_{\alpha}^{(+)} \sim d_{\alpha}, \\
\bar{N}_{\alpha} \bar{D}_{\alpha}^{(-)} \sim \nu_{\alpha}, & & \bar{N}_{\alpha} \bar{D}_{\alpha}^{(+)} \sim e_{\alpha} .
\end{array}
$$

We have four massless generations of right-handed quarks and leptons through the string-level Yukawa interactions of

$$
\begin{aligned}
W_{\text {right }} & =\sum_{i, \alpha} g_{i \alpha}^{\text {right-u}} \bar{u}_{i} \bar{C} \bar{D}^{(-)}+\sum_{i, \alpha} g_{i \alpha}^{\text {right-d }} \bar{d}_{i} \bar{C} \bar{D}^{(+)} \\
& +\sum_{i, \alpha} g_{i \alpha}^{\text {right }-\nu} \bar{\nu}_{i} \bar{N} \bar{D}^{(-)}+\sum_{i, \alpha} g_{i \alpha}^{\text {right }-\mathrm{e}} \bar{e}_{i} \bar{N} \bar{D}^{(+}(24)
\end{aligned}
$$

The detailed structure of the Yukawa coupling matrices $g_{i \alpha}^{\text {right-u}}$ and $g_{i \alpha}^{\text {right-d }}$ is also discussed in the next section. The values of masses are given as $g_{i \alpha}^{\text {right-u }} \Lambda_{R}$ and $g_{i \alpha}^{\text {right }-\mathrm{d}} \Lambda_{R}$ with the dynamical scale of $\operatorname{USp}(2)_{\mathrm{D} 6_{6}, \alpha}$. Here, 
we stress that the origin of generation in this model is not the multiple intersections of D6-branes, but the number of different D6-branes with the same winding numbers.

The similar happens in the intersecting $\mathrm{D} 6_{1}-\mathrm{D} 6_{3}-\mathrm{D} 6_{5}$ sector (Higgs sector). The confinement of two $\mathrm{USp}(2)_{\mathrm{D} 6_{5}, a}$ gauge interactions gives eight composite Higgs fields:

$$
T_{a} T^{(+)} \sim H_{a}^{(2)}, \quad T_{a} T^{(-)} \sim \bar{H}_{a}^{(1)},
$$

where $a=1,2,3,4$. Four of these eight composite Higgs fields become massive through the string-level Yukawa interactions of

$$
W_{\text {higgs }}=\sum_{i, a} g_{i a}^{(1)} H_{i}^{(1)} T_{a} T_{a}^{(-)}+\sum_{i, a} g_{i a}^{(2)} \bar{H}_{i}^{(2)} T_{a} T_{a}^{(+)} .
$$

The masses of four pairs of Higgs fields are given as $g_{i a}^{(1)} \Lambda_{H}$ and $g_{i a}^{(2)} \Lambda_{H}$, where $\Lambda_{H}$ is the scale of dynamics of $\operatorname{USp}(2)_{\mathrm{D} 6_{5}, a}$. Two pairs of composite Higgs fields remain massless.

From Fig 1 we see the existence of the following interactions.

$$
\begin{aligned}
W_{6-\text { fields }} & =\sum_{\alpha, \beta=1}^{6} \sum_{a=1}^{4} \frac{g_{\alpha \beta a}^{u}}{M_{s}^{3}}\left[C_{\alpha} D_{\alpha}\right]\left[\bar{C}_{\beta} \bar{D}_{\beta}^{(-)}\right]\left[T_{a} T_{a}^{(+)}\right] \\
& +\sum_{\alpha, \beta=1}^{6} \sum_{a=1}^{4} \frac{g_{\alpha \beta a}^{d}}{M_{s}^{3}}\left[C_{\alpha} D_{\alpha}\right]\left[\bar{C}_{\beta} \bar{D}_{\beta}^{(+)}\right]\left[T_{a} T_{a}^{(-)}\right] \\
& +\sum_{\alpha, \beta=1}^{6} \sum_{a=1}^{4} \frac{g_{\alpha \beta a}^{\nu}}{M_{s}^{3}}\left[N_{\alpha} D_{\alpha}\right]\left[\bar{N}_{\beta} \bar{D}_{\beta}^{(-)}\right]\left[T_{a} T_{a}^{(+)}\right] \\
& +\sum_{\alpha, \beta=1}^{6} \sum_{a=1}^{4} \frac{g_{\alpha \beta a}^{e}}{M_{s}^{3}}\left[N_{\alpha} D_{\alpha}\right]\left[\bar{N}_{\beta} \bar{D}_{\beta}^{(+)}\right]\left[T_{a} T_{a}^{(-)}\right\rceil 2
\end{aligned}
$$

After the "hypercolor" confinement these interactions give Yukawa interactions for the mass generation of quarks and leptons. The Yukawa coupling matrices are given by

$$
\begin{aligned}
& y_{\alpha \beta a}^{u} \simeq g_{\alpha \beta a}^{u} \frac{\Lambda_{L} \Lambda_{R} \Lambda_{H}}{M_{s}^{3}} \sim g_{\alpha \beta a}^{u}, \\
& y_{\alpha \beta a}^{d} \simeq g_{\alpha \beta a}^{d} \frac{\Lambda_{L} \Lambda_{R} \Lambda_{H}}{M_{s}^{3}} \sim g_{\alpha \beta a}^{d}, \\
& y_{\alpha \beta a}^{\nu} \simeq g_{\alpha \beta a}^{\nu} \frac{\Lambda_{L} \Lambda_{R} \Lambda_{H}}{M_{s}^{3}} \sim g_{\alpha \beta a}^{\nu}, \\
& y_{\alpha \beta a}^{e} \simeq g_{\alpha \beta a}^{e} \frac{\Lambda_{L} \Lambda_{R} \Lambda_{H}}{M_{s}^{3}} \sim g_{\alpha \beta a}^{e},
\end{aligned}
$$

since all the scales of dynamics, $\Lambda_{L}, \Lambda_{R}$ and $\Lambda_{H}$ are of the order of the string scale $M_{s}$. The detailed structure of these Yukawa coupling matrices $g_{\alpha \beta a}^{u}$ and $g_{\alpha \beta a}^{d}$ is investigated in the next section.

\section{The structure of Yukawa Coupling Matrices}

Here we investigate the structure of Yukawa coupling matrices for quark masses of the model introduced in the previous section. We do not require the realistic $\mu$-term (realistic value of the Higgs mass), since the aim of the analysis is simply to show the possibility of having nontrivial structure of Yukawa coupling matrices, e.g. nonvanishing mixing angles. The possibility of entire mass generation of quarks and leptons in this kind of models will be investigated in future works.

First, we evaluate the Yukawa coupling matrices $g_{i \alpha}^{\text {left }-\mathrm{q}}$ in Eq. (22) and $g_{i \alpha}^{\text {right }-\mathrm{u}}$ and $g_{i \alpha}^{\text {right-d }}$ in Eq.(24) by using Eq.(13). Three Yukawa-interacting fields are localized at three intersection points of these three D6-branes. The Yukawa couplings, $g_{i \alpha}^{\text {left-q }}, g_{i \alpha}^{\text {right }-\mathrm{u}}$ and $g_{i \alpha}^{\text {right-d }}$, are obtained as

$$
\begin{aligned}
g^{\text {left }-\mathrm{q}} & =\left(\begin{array}{cccccc}
\varepsilon_{3} & \varepsilon_{2} & 1 & \varepsilon_{1}^{2} \varepsilon_{2} & \varepsilon_{1}^{2} & \varepsilon_{1}^{2} \varepsilon_{2} \varepsilon_{3} \\
\varepsilon_{1}^{2} \varepsilon_{3} & \varepsilon_{1}^{2} \varepsilon_{2} & \varepsilon_{1}^{2} & \varepsilon_{2} & 1 & \varepsilon_{2} \varepsilon_{3}
\end{array}\right), \\
g^{\text {right }-\mathrm{u}} & =\left(\begin{array}{cccccc}
1 & \varepsilon_{2} & \varepsilon_{1} & \varepsilon_{2} \varepsilon_{3}^{2} & \varepsilon_{1} \varepsilon_{3}^{2} & \varepsilon_{1} \varepsilon_{2} \varepsilon_{3}^{2} \\
\varepsilon_{3}^{2} & \varepsilon_{2} \varepsilon_{3}^{2} & \varepsilon_{1} \varepsilon_{3}^{2} & \varepsilon_{2} & \varepsilon_{1} & \varepsilon_{1} \varepsilon_{2}
\end{array}\right), \\
g^{\text {right-d }} & =\left(\begin{array}{ccccccc}
\varepsilon_{1} & \varepsilon_{1} \varepsilon_{2} & 1 & \varepsilon_{1} \varepsilon_{2} \varepsilon_{3}^{2} & \varepsilon_{3}^{2} & \varepsilon_{2} \varepsilon_{3}^{2} \\
\varepsilon_{1} \varepsilon_{3}^{2} & \varepsilon_{1} \varepsilon_{2} \varepsilon_{3}^{2} & \varepsilon_{3}^{2} & \varepsilon_{1} \varepsilon_{2} & 1 & \varepsilon_{2}
\end{array}\right),
\end{aligned}
$$

where $\varepsilon_{i}=\exp \left(-A_{i} / 2 \pi \alpha^{\prime}\right)$ and $A_{i}$ is the $1 / 8$ of the area of the $i$-th torus. In general, classical solutions with larger area also contribute to the Yukawa couplings. However, we have used the approximation that the classical action corresponding to the minimum area contributes dominantly to the Yukawa couplings. In the following we assume that all $A_{i}$ are larger than $2 \pi \alpha^{\prime}$, namely $\varepsilon<1$. From these results we can say in good approximation that the massless left-handed quark doublets are $q_{1}, q_{2}, q_{4}$ and $q_{6}$, and the massless right-handed down-type quarks are $d_{1}, d_{2}, d_{4}$ and $d_{6}$. For right-handed up-type quarks, $u_{2}, u_{3}, u_{6}$ and one linear combination of $u_{4}$ and $u_{5}$ are approximately massless. Especially, in case of $\varepsilon_{1} \ll \varepsilon_{2}, u_{2}, u_{3}, u_{5}$ and $u_{6}$ are approximately massless, and in case of $\varepsilon_{2} \ll \varepsilon_{1}, u_{2}$, $u_{3}, u_{4}$ and $u_{6}$ are approximately massless.

Although it is not easy to calculate the 6-point coupling matrices of $g_{\alpha \beta a}^{u}$ and $g_{\alpha \beta a}^{d}$ in Eq.(27), generic n-point functions have been analyzed in Ref. 28. Our purpose is not to realize precisely realistic fermion masses and mixing angles, but to show that a new type of nontrivial Yukawa matrices can be derived in our scenario, e.g. suppressed but non-vanishing mixing angles. For such purpose, an approximation, which is reliable to order estimation on suppression factors, is sufficient. The six-point function consists of classical and quantum contributions, i.e. $Z_{c l} Z_{q}$, like the 3-point and 4-point functions. The classical part $Z_{c l}$ is important for our purpose because that can lead to a suppression factor, while the quantum part is expected to be of $\mathrm{O}(1)$ and does not contribute to lead to a suppression factor. The classical part is written as $Z_{c l}=\sum_{X_{c l}} e^{-S_{c l}}$ like Eq.(13), where $X_{c l}$ the classical string solutions, which have the asymptotic behavior corresponding to local open string modes near intersecting points. The minimum action corresponds to the sum of the areas of hexagons with six intersecting points on each torus [28. Hence, in order to give an order estimation of 6-point couplings $g_{\alpha \beta a}^{u, d}$, we use the approximation that $g_{\alpha \beta a}^{u, d} \sim \prod_{i} e^{-\Sigma_{i} / 2 \pi \alpha^{\prime}}$, which is the same as Eq.(13) except simply replacing the triangu- 
lar areas $\Sigma_{i}$ by the hexagonal areas. This approximation gives a sufficient order estimation of the exponential suppression enough for our purpose, though some sub-leading corrections are neglected. Actually, in our model, all the hexagons reduce to triangles, because some intersecting points always coincide and only three of the six sit at the different places. Hence, we obtain the following result.

$$
\begin{aligned}
& g_{\alpha \beta a=1}^{u}=g_{\alpha \beta a=1}^{d} \\
& =\left(\begin{array}{cccccc}
\varepsilon_{1} \varepsilon_{3} & \varepsilon_{1} \varepsilon_{2} \varepsilon_{3} & \varepsilon_{1}^{2} \varepsilon_{3} & \varepsilon_{1} \varepsilon_{2} \varepsilon_{3} & \varepsilon_{1}^{2} \varepsilon_{3} & \varepsilon_{1}^{2} \varepsilon_{2} \varepsilon_{3} \\
\varepsilon_{1} \varepsilon_{2} & \varepsilon_{1} \varepsilon_{2}^{2} & \varepsilon_{1}^{2} \varepsilon_{2} & \varepsilon_{1} \varepsilon_{2}^{2} \varepsilon_{3}^{2} & \varepsilon_{1}^{2} \varepsilon_{2} \varepsilon_{3}^{2} & \varepsilon_{1}^{2} \varepsilon_{2}^{2} \varepsilon_{3}^{2} \\
\varepsilon_{1} & \varepsilon_{1} \varepsilon_{2} & \varepsilon_{1}^{2} & \varepsilon_{1} \varepsilon_{2} \varepsilon_{3}^{2} & \varepsilon_{1}^{2} \varepsilon_{3}^{2} & \varepsilon_{1}^{2} \varepsilon_{2} \varepsilon_{3}^{2} \\
\varepsilon_{1} \varepsilon_{2} & \varepsilon_{1} \varepsilon_{2}^{2} & \varepsilon_{2} & \varepsilon_{1} \varepsilon_{2}^{2} \varepsilon_{3}^{2} & \varepsilon_{2} \varepsilon_{3}^{2} & \varepsilon_{2}^{2} \varepsilon_{3}^{2} \\
\varepsilon_{1} & \varepsilon_{1} \varepsilon_{2} & 1 & \varepsilon_{1} \varepsilon_{2} \varepsilon_{3}^{2} & \varepsilon_{3}^{2} & \varepsilon_{2} \varepsilon_{3}^{2} \\
\varepsilon_{1} \varepsilon_{2} \varepsilon_{3} & \varepsilon_{1} \varepsilon_{2}^{2} \varepsilon_{3} & \varepsilon_{2} \varepsilon_{3} & \varepsilon_{1} \varepsilon_{2}^{2} \varepsilon_{3} & \varepsilon_{2} \varepsilon_{3} & \varepsilon_{2}^{2} \varepsilon_{3}
\end{array}\right)_{\alpha \beta} \\
& g_{\alpha \beta a=2}^{u}=g_{\alpha \beta a=2}^{d} \\
& =\left(\begin{array}{cccccc}
\varepsilon_{2}^{2} \varepsilon_{3} & \varepsilon_{2} \varepsilon_{3} & \varepsilon_{1} \varepsilon_{2}^{2} \varepsilon_{3} & \varepsilon_{2} \varepsilon_{3} & \varepsilon_{1} \varepsilon_{2}^{2} \varepsilon_{3} & \varepsilon_{1} \varepsilon_{2} \varepsilon_{3} \\
\varepsilon_{2} & 1 & \varepsilon_{1} \varepsilon_{2} & \varepsilon_{3}^{2} & \varepsilon_{1} \varepsilon_{2} \varepsilon_{3}^{2} & \varepsilon_{1} \varepsilon_{3}^{2} \\
\varepsilon_{2}^{2} & \varepsilon_{2} & \varepsilon_{1} \varepsilon_{2}^{2} & \varepsilon_{2} \varepsilon_{3}^{2} & \varepsilon_{1} \varepsilon_{2}^{2} \varepsilon_{3}^{2} & \varepsilon_{1} \varepsilon_{2} \varepsilon_{3}^{2} \\
\varepsilon_{1}^{2} \varepsilon_{2} & \varepsilon_{1}^{2} & \varepsilon_{1} \varepsilon_{2} & \varepsilon_{1}^{2} \varepsilon_{3}^{2} & \varepsilon_{1} \varepsilon_{2} \varepsilon_{3}^{2} & \varepsilon_{1} \varepsilon_{3}^{2} \\
\varepsilon_{1}^{2} \varepsilon_{2}^{2} & \varepsilon_{1}^{2} \varepsilon_{2} & \varepsilon_{1} \varepsilon_{2}^{2} & \varepsilon_{1}^{2} \varepsilon_{2} \varepsilon_{3}^{2} & \varepsilon_{1} \varepsilon_{2}^{2} \varepsilon_{3}^{2} & \varepsilon_{1} \varepsilon_{2} \varepsilon_{3}^{2} \\
\varepsilon_{1}^{2} \varepsilon_{2} \varepsilon_{3} & \varepsilon_{1}^{2} \varepsilon_{3} & \varepsilon_{1} \varepsilon_{2} \varepsilon_{3} & \varepsilon_{1}^{2} \varepsilon_{3} & \varepsilon_{1} \varepsilon_{2} \varepsilon_{3} & \varepsilon_{1} \varepsilon_{3}
\end{array}\right)_{\alpha \beta} \\
& g_{\alpha \beta a=3}^{u}=g_{\alpha \beta a=3}^{d} \\
& =\left(\begin{array}{cccccc}
\varepsilon_{1} \varepsilon_{2}^{2} \varepsilon_{3} & \varepsilon_{1} \varepsilon_{2} \varepsilon_{3} & \varepsilon_{1}^{2} \varepsilon_{2}^{2} \varepsilon_{3} & \varepsilon_{1} \varepsilon_{2} \varepsilon_{3} & \varepsilon_{1}^{2} \varepsilon_{2}^{2} \varepsilon_{3} & \varepsilon_{1}^{2} \varepsilon_{2} \varepsilon_{3} \\
\varepsilon_{1} \varepsilon_{2} & \varepsilon_{1} & \varepsilon_{1}^{2} \varepsilon_{2} & \varepsilon_{1} \varepsilon_{3}^{2} & \varepsilon_{1}^{2} \varepsilon_{2} \varepsilon_{3}^{2} & \varepsilon_{1}^{2} \varepsilon_{3}^{2} \\
\varepsilon_{1} \varepsilon_{2}^{2} & \varepsilon_{1} \varepsilon_{2} & \varepsilon_{1}^{2} \varepsilon_{2}^{2} & \varepsilon_{1} \varepsilon_{2} \varepsilon_{3}^{2} & \varepsilon_{1}^{2} \varepsilon_{2}^{2} \varepsilon_{3}^{2} & \varepsilon_{1}^{2} \varepsilon_{2} \varepsilon_{3}^{2} \\
\varepsilon_{1} \varepsilon_{2} & \varepsilon_{1} & \varepsilon_{2} & \varepsilon_{1} \varepsilon_{3}^{2} & \varepsilon_{2} \varepsilon_{3}^{2} & \varepsilon_{3}^{2} \\
\varepsilon_{1} \varepsilon_{2}^{2} & \varepsilon_{1} \varepsilon_{2} & \varepsilon_{2}^{2} & \varepsilon_{1} \varepsilon_{2} \varepsilon_{3}^{2} & \varepsilon_{2}^{2} \varepsilon_{3}^{2} & \varepsilon_{2} \varepsilon_{3}^{2} \\
\varepsilon_{1} \varepsilon_{2} \varepsilon_{3} & \varepsilon_{1} \varepsilon_{3} & \varepsilon_{2} \varepsilon_{3} & \varepsilon_{1} \varepsilon_{3} & \varepsilon_{2} \varepsilon_{3} & \varepsilon_{3}
\end{array}\right)_{\alpha \beta} \\
& g_{\alpha \beta a=4}^{u}=g_{\alpha \beta a=4}^{d} \\
& =\left(\begin{array}{cccccc}
\varepsilon_{3} & \varepsilon_{2} \varepsilon_{3} & \varepsilon_{1} \varepsilon_{3} & \varepsilon_{2} \varepsilon_{3} & \varepsilon_{1} \varepsilon_{3} & \varepsilon_{1} \varepsilon_{2} \varepsilon_{3} \\
\varepsilon_{2} & \varepsilon_{2}^{2} & \varepsilon_{1} \varepsilon_{2} & \varepsilon_{2}^{2} \varepsilon_{3}^{2} & \varepsilon_{1} \varepsilon_{2} \varepsilon_{3}^{2} & \varepsilon_{1} \varepsilon_{2}^{2} \varepsilon_{3}^{2} \\
1 & \varepsilon_{2} & \varepsilon_{1} & \varepsilon_{2} \varepsilon_{3}^{2} & \varepsilon_{1} \varepsilon_{3}^{2} & \varepsilon_{1} \varepsilon_{2} \varepsilon_{3}^{2} \\
\varepsilon_{1}^{2} \varepsilon_{2} & \varepsilon_{1}^{2} \varepsilon_{2}^{2} & \varepsilon_{1} \varepsilon_{2} & \varepsilon_{1}^{2} \varepsilon_{2}^{2} \varepsilon_{3}^{2} & \varepsilon_{1} \varepsilon_{2} \varepsilon_{3}^{2} & \varepsilon_{1} \varepsilon_{2}^{2} \varepsilon_{3}^{2} \\
\varepsilon_{1}^{2} & \varepsilon_{1}^{2} \varepsilon_{2} & \varepsilon_{1} & \varepsilon_{1}^{2} \varepsilon_{2} \varepsilon_{3}^{2} & \varepsilon_{1} \varepsilon_{3}^{2} & \varepsilon_{1} \varepsilon_{2} \varepsilon_{3}^{2} \\
\varepsilon_{1}^{2} \varepsilon_{2} \varepsilon_{3} & \varepsilon_{1}^{2} \varepsilon_{2}^{2} \varepsilon_{3} & \varepsilon_{1} \varepsilon_{2} \varepsilon_{3} & \varepsilon_{1}^{2} \varepsilon_{2}^{2} \varepsilon_{3} & \varepsilon_{1} \varepsilon_{2} \varepsilon_{3} & \varepsilon_{1} \varepsilon_{2}^{2} \varepsilon_{3}
\end{array}\right)_{\alpha \beta}
\end{aligned}
$$

Since we have six pair of Higgs fields and only the composite Higgs fields have Yukawa couplings, there are eight Yukawa coupling matrices for quark masses.

All of these Yukawa matrices are nontrivial in the sense that they differ from the factorizable form of Eq.(15). Detail calculation is necessary for full analysis on these Yukawa matrices by varying parameters. However, our purpose is not to analyze this model in detail, but to show the possibility for leading to nontrivial results in our scenario, i.e. non-vanishing mass ratios and mixing angles, which can be realistic. Therefore, here we show an simple case.

Now, let us consider the case with $\varepsilon_{2} \ll \varepsilon_{1}$. In this case, the modes $u_{1}$ and $u_{5}$ as well as $q_{3}, q_{5}, d_{3}$ and $d_{5}$ dominantly gain large masses through the interactions of Eqs.(22) and (24) with coupling matrices of Eqs.(32), (33) and (34). The other modes approximately correspond to four generations. For example, let us consider the Yukawa matrices, $g_{a=2}^{u}$ and $g_{a=3}^{d}$, that is, we assume that one pair of com- posite Higgs doublet fields, $\left(\bar{H}_{3}^{(1)}, H_{2}^{(2)}\right)$, is dominant to generate quark mass matrices at the weak scale. Then we consider the parameter region that $\varepsilon_{2} \ll \varepsilon_{3} \ll \varepsilon_{1}(\sim 1)$, and in this parameter region the matter fields $\left(q_{2}, q_{4}, q_{6}\right)$, $\left(u_{2}, u_{6}\right)$ and $\left(d_{2}, d_{6}\right)$ have large entries in the Yukawa matrices $g_{a=2}^{u}$ and $g_{a=3}^{d}$. Here, let us concentrate ourselves to two heavy modes among four generations. The $(2 \times 2)$ submatrices with large entries in the Yukawa matrices $g_{a=2}^{u}$ and $g_{a=3}^{d}$ are written up to $O\left(\varepsilon_{3}^{2}\right)$ as

$$
g_{a=2}^{u} \rightarrow\left(\begin{array}{cc}
1 & 0 \\
\varepsilon_{1}^{2} \varepsilon_{3} & \varepsilon_{1} \varepsilon_{3}
\end{array}\right)
$$

for the up sector,

$$
g_{a=3}^{d} \rightarrow\left(\begin{array}{cc}
\varepsilon_{1} & 0 \\
\varepsilon_{1} \varepsilon_{3} & \varepsilon_{3}
\end{array}\right)
$$

for the down sector in the field basis $\left(\left(q_{2}+q_{4}\right) / \sqrt{2}, q_{6}\right)$, $\left(u_{2}, u_{6}\right),\left(d_{2}, d_{6}\right)$.

Now we can calculate mass eigenvalues of two heavy modes among four generations, i.e. $m_{u, 3}$ and $m_{u, 4}$ for the up sector and $m_{d, 3}$ and $m_{d, 4}$ for the down sector, and their mixing angle $V_{34}$. The mass ratios and the mixing angle is obtained as

$$
\frac{m_{u, 3}}{m_{u, 4}} \approx \varepsilon_{1} \varepsilon_{3}, \quad \frac{m_{d, 3}}{m_{d, 4}} \approx \frac{\varepsilon_{3}}{\varepsilon_{1}}, \quad V_{34} \approx \varepsilon_{3},
$$

that is, we have the following relation,

$$
\sqrt{\frac{m_{u, 3}}{m_{u, 4}} \frac{m_{d, 3}}{m_{d, 4}}} \approx V_{34}
$$

at the composite scale.

It is interesting to compare these results with the experimental values of quark mass ratios, $\frac{m_{c}}{m_{t}}$ and $\frac{m_{s}}{m_{b}}$, and the mixing angle $V_{c b}$. At the weak scale, the experimental values of mass ratios, $\frac{m_{c}}{m_{t}}=0.0038$ and $\frac{m_{s}}{m_{b}}=0.025$, lead to

$$
\sqrt{\frac{m_{c}}{m_{t}} \frac{m_{s}}{m_{b}}}=0.01
$$

and the mixing angle is

$$
V_{c b}=0.04
$$

We find that the values of parameters $\varepsilon_{1} \sim 0.5$ and $\varepsilon_{3} \sim$ 0.01 lead to almost realistic structure of quark Yukawa coupling matrices. Note that our approximation is reliable as order estimation.

We have shown that the structure of the Yukawa coupling matrices at least for the $(2 \times 2)$ sub-matrices can be realistic in the simple case through our scenario of the dynamical generation of Yukawa coupling matrices. The most relevant fact is the different origin of the generation from that in the conventional intersecting D-brane models. The origin of the generation is not the multiple intersection of D-branes, but many different D-branes with the same multiplicity and the same winding numbers.

We give several comments in order before closing this section. 
In this model a large Yukawa coupling is obtained in case of which all six localization points of "preons" are coincides in all three tori. Therefore, it seems very difficult to obtain the Yukawa coupling of the order of unity. But, we have shown that it is possible and can accidentally happen (see Eq. (39)).

For more rigorous investigations, we should take care of the normalization of fields. The low-energy effective fields in string theory should be normalized by considering the moduli dependence of the Kähler potential. A concrete analysis on the moduli dependence of the Kähler potential in type IIA intersecting D-brane models is given in Ref. 20]. The normalization of the low-energy effective field after "hypercolor" confinement should also be considered more precisely. Unfortunately, there is no established method to obtain the Kähler potentials for composite fields in strong coupling gauge theories in four dimensions, except for some special cases 29 30.

\section{Conclusions}

The structure of Yukawa coupling matrices in models from type IIA $T^{6} /\left(\mathrm{Z}_{2} \times \mathrm{Z}_{2}\right)$ orientifolds $\left(T^{6}=T^{2} \times T^{2} \times T^{2}\right)$ with intersecting D-branes has been investigated. There is a difficulty to have a realistic Yukawa coupling matrices in the models in which the generation structure of quarks and leptons is originated from the multiple intersection of D-branes. On the other hand, it has been shown that the structure of Yukawa coupling matrices in the models with dynamical generation of Yukawa coupling matrices is nontrivial. Indeed, realistic values of the mixing angles $V_{c b}$ and mass ratios $m_{c} / m_{t}$ and $m_{s} / m_{b}$ can be realized. The most relevant fact is the different origin of the generation. The origin of the generation is not the multiple intersection of D-branes, but many different D-branes with the same multiplicity and the same winding numbers.

Here we give a comment on the origin of $\mathrm{CP}$ violating phases. Inclusion of Wilson lines is one source of $\mathrm{CP}$ phases in Yukawa matrices [17]. As another source, the holomorphic dynamical scales $\Lambda_{R, L, H}$ may provide CP phases in effective Yukawa couplings in this class of models.

Although the possibility of having realistic Yukawa coupling matrices, this class of models with dynamical generation of Yukawa coupling matrices have many other phenomenological issues to be solved: constructing models with three generations and light Higgs pairs with realistic $\mu$-term for electroweak symmetry breaking, realization of the supersymmetry breaking, and so on. In addition to these which are common in all the supersymmetric type IIA intersecting D-brane models, composite models usually have a problem of rapid proton decay. It would be very interesting to explorer more realistic models by incorporating the results of efforts of the model buildings based on the field theory into the framework of the intersecting D-brane model.

The authors would like to thank Tatsuya Noguchi for useful discussions. N. K., T. K. and N. O. would like to thank hospitality of RIKEN, where a part of this work was studied. T. K. is supported in part by the Grant-in-Aid for Scientific Research (\#16028211) and the Grant-in-Aid for the 21st Century COE "The Center for Diversity and Universality in Physics" from the Ministry of Education, Culture, Sports, Science and Technology of Japan. N. M. is supported by the Special Postdoctoral Researchers Program at RIKEN. N. O. is supported in part by the Grant-in-Aid for Scientific Research (\#15740164).

\section{References}

1. R. Blumenhagen, L. Görlich, B. Körs and D. Lüst, JHEP 0010 (2000) 006, hep-th/0007024

2. G. Aldazabal, S. Franco, L.E. Ibáñez, R. Rabadán and A. M. Uranga, J. Math. Phys. 42 (2001) 3103, hep-th/0011073 JHEP 0102 (2001) 047, hep-ph/0011132

3. R. Blumenhagen, B. Körs and D. Lüst, JHEP 0102 (2001) 030, hep-th/0012156

4. M. Cvetič, G. Shiu and A.M. Uranga, Phys. Rev. Lett. 87 (2001) 201801, hep-th/0107143 Nucl. Phys. B 615 (2001) 3, hep-th/0107166

5. S. Förste, G. Honecker and R. Schreyer, Nucl. Phys. B 593 (2001) 127, hep-th/0008250

6. R. Blumenhagen, L. Görlich and T. Ott, JHEP 0301 (2003) 021, hep-th/0211059

7. M. Cvetič, I. Papadimitriou and G. Shiu, Nucl. Phys. B 659 (2003) 193, hep-th/0212177

8. G. Honecker, Nucl. Phys. B 666 (2003) 175, hep-th/0303015

9. M. Larosa and G. Pradisi, Nucl. Phys. B 667 (2003) 261, hep-th/0305224

10. M. Cvetič and I. Papadimitriou, D 67 (2003) 126006, hep-th/0303197

11. N. Kitazawa, Nucl. Phys. B 699 (2004) 124, hep-th/0401096

12. M. Cvetič, T. Li and T. Liu, Nucl. Phys. B 698 (2004) 163, hep-th/0403061

13. G. Honecker and T. Ott, hep-th/0404055

14. M. Cvetič, P. Langacker and G. Shiu, Nucl. Phys. B 642 (2002) 139, hep-th/0206115

15. N. Kitazawa, JHEP 0411 (2004) 044, hep-th/0403278

16. M. Berkooz, M. R. Douglas and R. G. Leigh, Nucl. Phys. B 480 (1996) 265, hep-th/9606139

17. D. Cremades, L.E. Ibáñez and F. Marchesano, JHEP 0307 (2003) 038, hep-th/0302105

18. M. Cvetič and I. Papadimitriou, Phys. Rev. D 68 (2003) 046001, hep-th/0303083

19. S. A. Abel and A. W. Owen, Nucl. Phys. B 663 (2003) 197, hep-th/0303124

20. D. Lüst, P. Mayr, R. Richter and S. Stieberger, Nucl. Phys. B 696 (2004) 205, hep-th/0404134

21. D. Cremades, L. E. Ibáñez and F. Marchesano, hep-th/0404229

22. L. J. Dixon, D. Friedan, E. J. Martinec and S. H. Shenker, Nucl. Phys. B 282 (1987) 13; S. Hamidi and C. Vafa, Nucl. Phys. B 279 (1987) 465.

23. T. T. Burwick, R. K. Kaiser and H. F. Müller, Nucl. Phys. B 355 (1991) 689. J. Erler, D. Jungnickel, M. Spaliński and S. Stieberger, Nucl. Phys. B 397 (1993) 379, hep-th/9207049

24. T. Kobayashi and O. Lebedev, Phys. Lett. B 566 (2003) 164, hep-th/0303009 Phys. Lett. B 565193 (2003) 193, hep-th/0304212 
25. P. Ko, T. Kobayashi and J. h. Park, Phys. Lett. B 598 (2004) 263, hep-ph/0406041

26. See for Yukawa couplings in non-supersymmetric models, e.g., S. A. Abel, O. Lebedev and J. Santiago, hep-ph/0312157

27. N. Chamoun, S. Khalil and E. Lashin, Phys. Rev. D 69 (2004) 095011, hep-ph/0309169

28. S. A. Abel and A. W. Owen, Nucl. Phys. B 682 (2004) 183, hep-th/0310257

29. I. Affleck, M. Dine and N. Seiberg, Nucl. Phys. B 256 (1985) 557.

30. E. Poppitz and L. Randall, Phys. Lett. B336, 402 (1994).

Phys. Lett. B 336 (1994) 402, hep-th/9407185 